\title{
Joint Angle-Amplitude Estimation for Multiple Signals with L-Structured Arrays Using Bioinspired Computing
}

\author{
Fawad Zaman \\ Department of Electrical Engineering, COMSATS Institute of Information Technology, Attock Campus, Punjab, Pakistan \\ Correspondence should be addressed to Fawad Zaman; fawad@ciit-attock.edu.pk
}

Received 28 July 2016; Revised 10 October 2016; Accepted 24 October 2016; Published 15 January 2017

Academic Editor: Donatella Darsena

Copyright (C) 2017 Fawad Zaman. This is an open access article distributed under the Creative Commons Attribution License, which permits unrestricted use, distribution, and reproduction in any medium, provided the original work is properly cited.

\begin{abstract}
The aim of this work is to estimate jointly the elevation and azimuth angles along with the amplitudes of multiple signals impinging on 1-L- and 2-L-shape arrays. An efficient mechanism based on hybrid Bioinspired techniques is proposed for this purpose. The global search optimizers such as Differential Evolution (DE) and Particle Swarm optimization (PSO) are hybridized with a local search optimizer called pattern search (PS). Approximation theory in Mean Square Error sense is exploited to develop a fitness function of the problem. The unknown parameters of multiple signals transmitted by far-field sources are estimated with the strength of hybrid DE-PS and PSO-PS. The effectiveness of the proposed techniques is tested in terms of estimation accuracy, proximity effect, convergence, and computational complexity.
\end{abstract}

\section{Introduction}

Parameter estimation such as Direction of Arrival (DOA) of electromagnetic signals for multiple sources is one of the vital areas of research in array signal processing from the last few decades. The DOA estimation ensues in adaptive beamforming to place the nulls in the direction of jammers or unwanted signals, while placing the main beam in the desired direction [1-3]. Therefore, various schemes are investigated for DOA estimation in literature but mostly limited to one-dimensional (1D) DOA estimation of sources impinging on linear array [4-6]. The 1D DOA estimation is comparatively straight forward because it only involves the angle of elevation as function of DOA. However, 2D DOA estimation is relatively complicated as it involves both elevation and azimuth angles [7-11]. 2D DOA estimation has engaged the interest of research community in the field of radar, sonar, wireless communication systems, and so forth [12]. The estimation failure, pair matching between elevation and azimuth angles, and computational complexity are the major problems observed in 2D DOA estimation. The different array configurations have been proposed to address the 2D DOA estimation problem [13-15] but lack the requisite resources to resolve the aforementioned problems. In [16],
Propagator Method (PM) with parallel shape array is used to overcome the computational complexity of [15], but the estimation failure and pair matching problems still exist. In [17], the PM is applied with L-shape array to surmount the drawbacks of [16] but requires a large number of snapshots and sensors.

In the present scientific society era, the significance of Evolutionary Computing Techniques (ECT) that include Genetic Algorithm (GA), Particle Swarm Optimization (PSO), and Differential Evolution (DE) cannot be vilipended. These techniques are not only easy to implement but also have the significant ability of hybridization with other heuristic and nonheuristic techniques. Therefore, the significance of these techniques is realized in different varying nature of applications [18-22].

In this paper, DE and PSO are hybridized with PS to jointly estimate the amplitude and 2D DOA of far-field sources impinging on 1-L- and 2-L-shape arrays. In this hybridization process, DE and PSO act as global search optimizers, while PS is used as rapid local search optimizer. The best individual results of DE and PSO are given to PS for further tuning. The performance criterion is devised on the basis of Mean Square Error (MSE) that is applied as an objective evaluation function. This fitness function is 


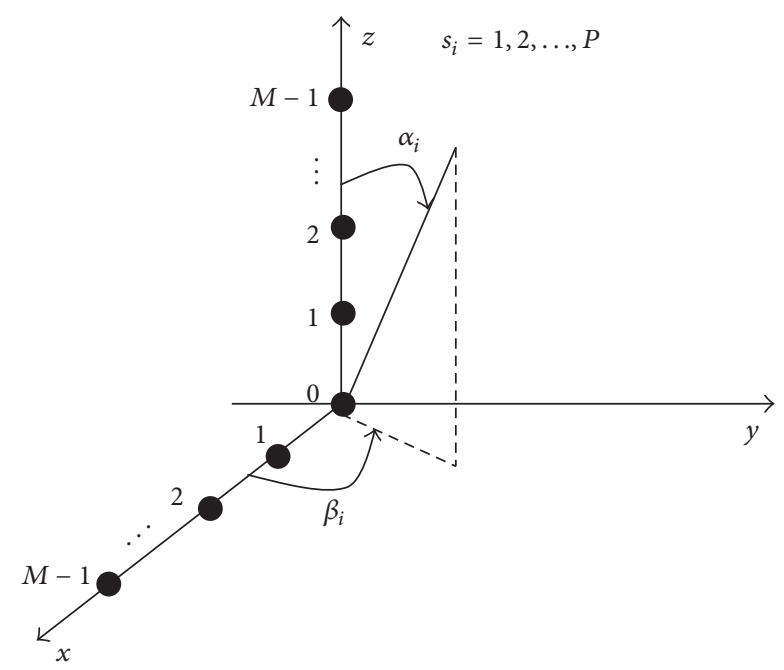

FIGURE 1: 1-L-shape array having 2 subarrays.

derived from Maximum Likelihood Principle (MLP) [21]. The interest behind this objective function is ease in concept, ease in implementation, and robustness against noise and requires single snapshot. The performance of hybrid (DEPS) and hybrid (PSO-PS) is first compared with DE and PSO techniques. Moreover, the results are also compared with the existing PM, which uses parallel [16] and L-shape arrays [17].

The rest of the paper is organized as follows. Section 2 formulates the problem, while Section 3 describes the proposed methodology structure. In Section 4 results and discussion are provided, while Section 5 is dedicated for conclusion and future work directions.

\section{Data Model}

In this section, data model is developed for $P$ independent sources impinging on 1-L- and 2-L-shape arrays from far field. The 1-L-shape array consists of two subarrays, which are placed along $x$-axis and $z$-axis. The 2 -L-shape array is similar to 1-L-shape array except that it has an extra subarray along $y$-axis. The number of antenna elements in each subarray is $M-1$, while the reference element is common for all subarrays as shown in Figures 1 and 2. The distance " $d$ " between two consecutive elements in each subarray is $\lambda / 2$. All the impinging signals are considered to be narrow band with known frequency $\left(\omega_{0}\right)$ by having different amplitudes $\left(s_{i}\right)$, elevation angles $\left(\alpha_{i}\right)$, and azimuth angles $\left(\beta_{i}\right)$, where $i=1,2, \ldots, P$.

(a) 1-L-Shape Array. The output at $l$ th sensor of $z$-axis subarray due to $P$ sources is given as

$$
y_{z l}(1 \mathrm{~L})=\sum_{i=1}^{P} a_{z l}\left(\alpha_{i}\right) s_{i}+n_{z l} .
$$

In $(1), y_{z l}(1 \mathrm{~L})$ means the response of $l$ th element placed in $z$-axis subarray of 1-L-shape array, while

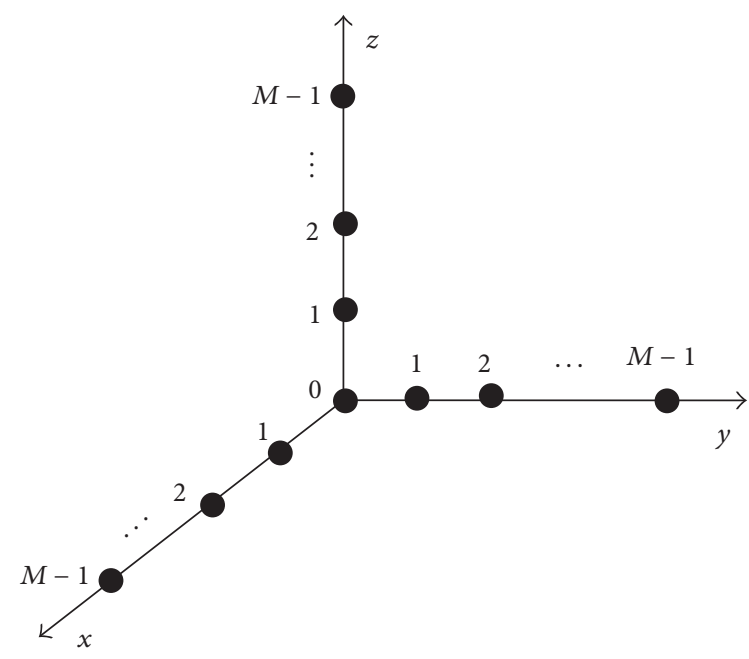

FIGURE 2: 2-L-shape array having 3 subarrays.

$a_{z l}\left(\alpha_{i}\right)=\exp \left(-j\left(2 \pi l d \cos \alpha_{i} / \lambda\right)\right)$ and, for $d=\lambda / 2$ and $l=$ $0,1,2, \ldots, M-1$, the output of complete subarray can be given as

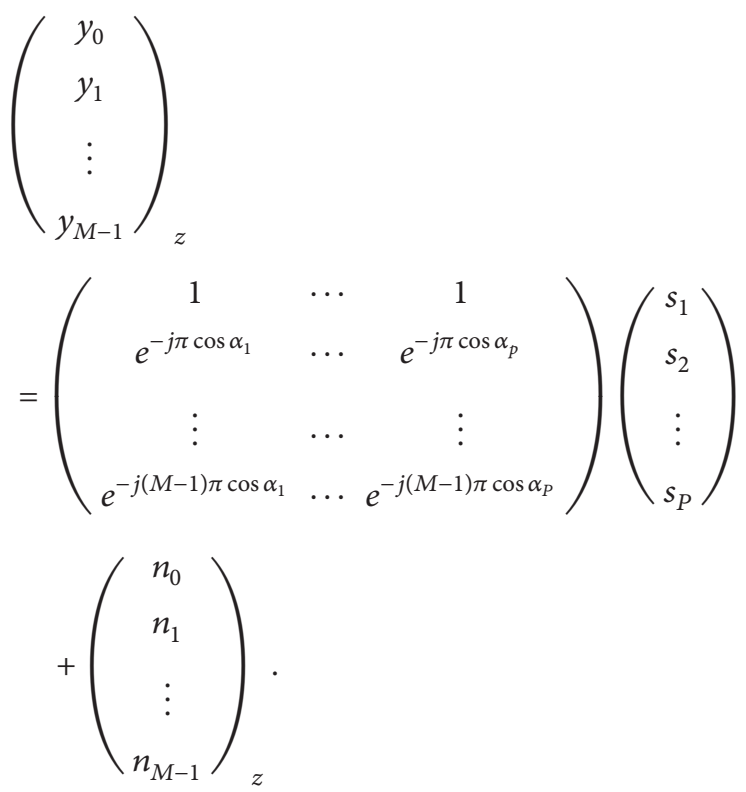

Generally, it can be written as

$$
\mathbf{y}_{z}=\mathbf{A}_{z}(\alpha) \mathbf{s}+\mathbf{n}_{z}
$$

In (3), $\mathbf{A}_{z}$ is called steering matrix which contains the elevation angles of the received signals, while $\mathbf{n}_{z}$ is an Additive White Gaussian Noise (AWGN) added at the output of each sensor along $z$-axis. In the same way, the response of $x$-axis subarray for $l$ th sensor can be given as

$$
y_{x l}(1 \mathrm{~L})=\sum_{i=1}^{P} a_{x l}\left(\alpha_{i}, \beta_{i}\right) s_{i}+n_{x l} .
$$


In (4), $a_{x l}\left(\alpha_{i}, \beta_{i}\right)=\exp \left(-j\left(2 \pi l d \cos \alpha_{i} \cos \beta_{i} / \lambda\right)\right)$ and, for $d=$ $\lambda / 2$ and $l=0,1,2, \ldots, M-1$, we have

$$
\begin{aligned}
& \left(\begin{array}{c}
y_{0} \\
y_{1} \\
\vdots \\
y_{M-1}
\end{array}\right)_{x} \\
& =\left(\begin{array}{ccc}
e^{-j \pi \sin \alpha_{1} \cos \beta_{1}} & \ldots & e^{-j \pi \sin \alpha_{P} \cos \beta_{P}} \\
\vdots & \ldots & \vdots \\
e^{-j(M-1) \pi \sin \alpha_{1} \cos \beta_{1}} & \cdots & e^{-j(M-1) \pi \sin \alpha_{P} \cos \beta_{P}}
\end{array}\right)\left(\begin{array}{c}
s_{1} \\
s_{2} \\
\vdots \\
s_{P}
\end{array}\right) \\
& +\left(\begin{array}{c}
n_{0} \\
n_{1} \\
\vdots \\
n_{M-1}
\end{array}\right) .
\end{aligned}
$$

Generally,

$$
\mathbf{y}_{x}=\mathbf{A}_{x}(\alpha, \beta) \mathbf{s}+\mathbf{n}_{x}
$$

(b) 2-L-Shape Array. In case of 2-L-shape array, the output of $x$-axis and $z$-axis subarrays is exactly similar as discussed for 1-L-shape array; however, the output of $y$-axis subarray at $l$ th sensor can be modeled as

$$
y_{y l}(2 \mathrm{~L})=\sum_{i=1}^{P} a_{y l}\left(\alpha_{i}, \beta_{i}\right) s_{i}+n_{y l} .
$$

In $(7), a_{y l}\left(\alpha_{i}, \beta_{i}\right)=\exp \left(-j\left(2 \pi l d \sin \alpha_{i} \sin \beta_{i} / \lambda\right)\right)$, and

$$
\begin{aligned}
& \left(\begin{array}{c}
y_{0} \\
y_{1} \\
\vdots \\
y_{M-1}
\end{array}\right)_{y}{ }^{1} \\
& =\left(\begin{array}{ccc}
e^{-j \pi \sin \alpha_{1} \sin \beta_{1}} & \ldots & e^{-j \pi \sin \alpha_{P} \sin \beta_{P}} \\
\vdots & \ldots & \vdots \\
e^{-j(M-1) \pi \sin \alpha_{1} \sin \beta_{1}} & \cdots & e^{-j(M-1) \pi \sin \alpha_{P} \sin \beta_{P}}
\end{array}\right)\left(\begin{array}{c}
s_{1} \\
s_{2} \\
\vdots \\
s_{P}
\end{array}\right) \\
& +\left(\begin{array}{c}
n_{0} \\
n_{1} \\
\vdots \\
n_{M-1}
\end{array}\right), \\
& \mathbf{y}_{y}=\mathbf{A}_{y}(\alpha, \beta) \mathbf{s}+\mathbf{n}_{y} .
\end{aligned}
$$

From (2), (5), and (8), it is clear that the unknown signal parameters are amplitudes $\left(s_{i}\right)$, elevation angles $\left(\alpha_{i}\right)$, and azimuth angles $\left(\beta_{i}\right)$ to be estimated for $i=1,2, \ldots, P$.
TABLE 1: Parameter setting for PS.

\begin{tabular}{lc}
\hline Parameters & Setting \\
\hline Starting point & The best particle achieved through PSO or DE \\
Poll method & GPS positive basis 2N \\
Polling order & Consecutive \\
Maximum iteration & 800 \\
Function evaluation & 15000 \\
Mesh size & 01 \\
Expansion factor & 2.0 \\
Contraction factor & 0.5 \\
Penalty factor & 100 \\
Bind tolerance & $10-07$ \\
Mesh tolerance & $10-09$ \\
X tolerance & $10-09$ \\
\hline
\end{tabular}

\section{Proposed Techniques}

In this section, we have discussed the brief introduction, flow chart, parameter setting, and pseudocode of PS, PSO, and DE.

3.1. Pattern Search. Pattern Search (PS) which is also called direct search method belongs to a family of numerical optimization techniques. It can be used for linearly constrained and bounded optimization problem, which does not require the gradient of the problem. PS works on a group of points called a pattern. If improvement does not occur in the objective function at the current iteration, the pattern is refined and the process is repeated [23].

The PS acts even well when it is used as a local search optimizer with any other global optimizer technique such as GA, PSO, and DE. In the present work, PS is used as a local search optimizer with DE and PSO. The best particle achieved through DE and PSO is given as starting point to PS for further tuning. For PS, we have used a MATLAB builtin optimization tool box for which the parameters settings are given in Table 1.

3.2. Particle Swarm Optimization (PSO). The idea of PSO was first introduced by Kennedy and Eberhart being inspired from the group of birds flocking for food in random manner [24]. It is an Evolutionary Computing Technique (ECT) based on iterative process in order to obtain the best optimization. The idea to search for food is heuristic because all the birds know their distance from the food but do not actually know the location. They find their food by sharing their search information in cooperative behavior in contrast to GA by crossover and kids production method. The PSO has acquired applications almost in every field of engineering [25, 26]. In the current work, PSO is used as global optimizer and the results of PSO are further provided to PS for substantial improvement. For both L-shape arrays, the implementation of PSO is quite similar with some instance of divergence. The generic flow diagram for PSO is shown in Figure 3(a), 


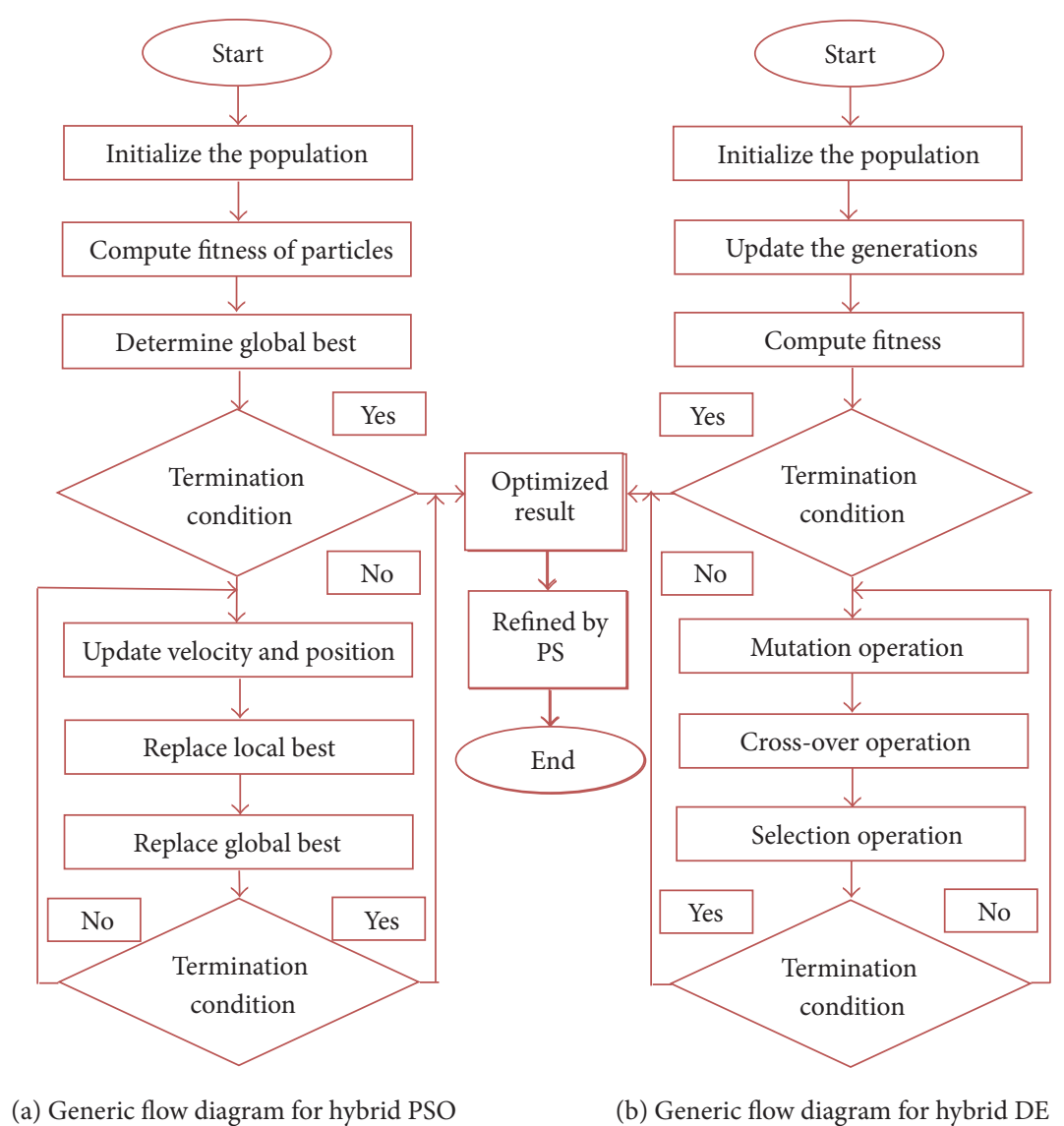

Figure 3

whereas the steps involved in the implementation of PSO are characterized in the form of pseudocodes.

Step 1 (initialization). Like other ECT, the first step is to produce randomly $N$ number of particles. The length of each particle depends on the number of the unknowns in the problem. In the present case, the length of each particle is $3 \times P$, where $P$ is the total number of sources. The first $P$ genes in each particle represent elevation angles and the next $P$ genes represent azimuth angles, while the last $P$ genes correspond to the amplitudes of sources. The particles in case of 1-L-shape array are produced as

$$
\mathbf{C}=\left(\begin{array}{ccc}
\alpha_{1,1} \alpha_{1,2} \cdots \alpha_{1, P} & \beta_{1, P+1} \beta_{1, P+2} \cdots \beta_{1,2 P} & s_{1,2 P+1} s_{1,2 P+2} \cdots s_{1,3 P} \\
\alpha_{2,1} \alpha_{2,2} \cdots \alpha_{2, P} & \beta_{2, P+1} \beta_{2, P+2} \cdots \beta_{2,2 P} & s_{2,2 P+1} s_{2,2 P+2} \cdots s_{2,3 P} \\
\vdots & \vdots & \vdots \\
\alpha_{N, 1} \alpha_{N, 2} \cdots \alpha_{N, P} & \beta_{N, P+1} \beta_{N, P+2} \cdots \beta_{N, 2 P} & s_{N, 2 P+1} s_{N, 2 P+2} \cdots s_{N, 3 P}
\end{array}\right)
$$

In the same way, $N$ number of random particles for 2-L-shape array can be generated. The major difference between the particles of 1-L- and 2-L-shape array is the range of elevation angle. For 1-L-shape array, the genes of elevation angles are generated from 0 to $\pi / 2$, while, for 2 -L-shape array, the genes are produced for the range of elevation angles from 0 to $\pi$. The range of amplitudes and azimuth angles are the same for both L-shape arrays. The lower and upper ranges of $\alpha, \beta$, and $s$ are defined as

$$
\alpha_{i, k}(1 \mathrm{~L}) \in R: 0 \leq \alpha_{i, k} \leq \frac{\pi}{2}
$$

$$
\begin{aligned}
& \alpha_{i, k}(2 \mathrm{~L}) \in R: 0 \leq \alpha_{i, k} \leq \pi \\
& \beta_{i, P+k} \in R: 0 \leq \beta_{i, P+k} \leq 2 \pi \\
& s_{i, 2 P+k} \in R: L_{b} \leq s_{i, 2 P+k} \leq U_{b} \\
& \quad \text { for, } i=1,2, \ldots, N, k=1,2, \ldots, P,
\end{aligned}
$$

where $L_{b}$ and $U_{b}$ represent the lower and upper bounds of signals amplitude. 
Step 2 (fitness function). MSE is used as fitness evaluation function for both L-shape arrays which can be given as

$$
\mathrm{FF}(i)=\frac{1}{(1+\zeta(i))},
$$

where $\zeta(i)$ defines the difference function for $i$ th particle. For 1-L-shape array it can be given as

$$
\zeta=\frac{1}{2 M}\left(\zeta_{1}+\zeta_{2}\right)
$$

For 2-L-shape array it can be defined as

$$
\zeta=\frac{1}{3 M}\left(\zeta_{1}+\zeta_{2}+\zeta_{3}\right) .
$$

In (13) and (14),

$$
\begin{aligned}
& \zeta_{1}(i)=\sum_{l=0}^{M-1}\left|y_{z l}-\widehat{y}_{z l}^{i}\right|^{2}, \\
& \zeta_{2}(i)=\sum_{l=0}^{M-1}\left|y_{x l}-\hat{y}_{x l}^{i}\right|^{2}, \\
& \zeta_{3}(i)=\sum_{l=0}^{M-1}\left|y_{y l}-\hat{y}_{y l}^{i}\right|^{2} .
\end{aligned}
$$

In (15), $y_{z l}, y_{x l}, y_{y l}$ are defined in (1), (2), and (5) respectively, while $\widehat{y}_{z l}^{i}, \widehat{y}_{x l}^{i}, \widehat{y}_{y l}^{i}$ are defined as

$$
\begin{aligned}
& \hat{y}_{z l}^{i}=\sum_{k=1}^{P} \exp \left(-j \pi l \cos \left(c_{k}^{i}\right)\right) \cdot c_{2 P+k}^{i}, \\
& \widehat{y}_{x l}^{i}=\sum_{k=1}^{P} \exp \left(-j \pi l \sin \left(c_{k}^{i}\right) \cos \left(c_{P+k}^{i}\right)\right) \cdot c_{2 P+k}^{i}, \\
& \widehat{y}_{y l}^{i}=\sum_{k=1}^{P} \exp \left(-j \pi l \sin \left(c_{k}^{i}\right) \sin \left(c_{P+k}^{i}\right)\right) \cdot c_{2 P+k}^{i},
\end{aligned}
$$

where $c^{i}$ is defined in (10). The particle as global best $\mathbf{g}_{b}$ is stored that has maximum fitness function, while marking each $\mathbf{c}_{i}$ as local best $\mathbf{l}_{i}$ for this step where $i=1,2, \ldots, N$.

Step 3 (termination criterion). If the desired fitness function is achieved, stop and go to Step 7; otherwise go to Step 4a.

Step $4 a$ (updating particle velocity). To update the velocity of each particle generated for both L-shape arrays, we used the following relation:

$$
\begin{aligned}
v_{i k}(n)= & v_{i k}(n-1)+\phi_{1}(1-\gamma)\left(l_{i k}-c_{i k}(n-1)\right) \\
& +\phi_{2} \gamma\left(g_{b}-c_{i k}(n-1)\right),
\end{aligned}
$$

where $\phi_{1}=\phi_{2}=1$ while $0.1 \leq \gamma \leq 0.9$. Initially, the value of $\gamma$ is taken as 0.1 and then gradually increases from 0.1 to 0.9 . Therefore, in the beginning more weight factor or ratio is given to local intelligence, whereas at the end more importance is provided to collective intelligence. Hence, the velocity is doubly bounded

$$
\begin{aligned}
& -v_{\max } \leq v_{i k} \leq v_{\max }, \\
& \text { If } v_{i k}(n)>v_{\max } \text {, } \\
& v_{i k}(n)=v_{\max }, \\
& \text { if } \quad v_{i k}(n)<-v_{\max } \text {, } \\
& v_{i k}(n)=-v_{\max } \text {. }
\end{aligned}
$$

Step $4 b$ (updating position). To update the position of particles, the following relation is used:

$$
\mathbf{c}_{i k}(n)=\mathbf{c}_{i k}(n-1)+\mathbf{v}_{i k}(n)
$$

Step $5 a$ (choosing local best particle). The previous local best $\mathbf{l}(n)$ is replaced with $\mathbf{c}(n)$, if fitness of $\mathbf{c}(n)>$ fitness of $\mathbf{l}(n)$.

Step $5 b$ (choosing global best particle). Also replace $\mathbf{g}_{b}$ with $\mathbf{c}(n)$ as new global best if fitness of $\mathbf{c}(n)>\mathbf{g}_{b}$.

Step 6 (termination). The termination criteria are based on the achievement of the following goals:

(i) The required fitness function is achieved.

(ii) The desired MSE has been reached which is $10^{-7}$.

(iii) The total number of iterations has been completed.

Step 7 (hybridization). In this step, the best values of amplitude and DOA got for both L-shape arrays are given as starting point to PS for further refinement. The parameter setting for PS is provided in Table 1.

Step 8 (storage). Store all the results for later in discussion and comparison.

3.3. Differential Evolution (DE). The Differential Evolution (DE) was first introduced by Stone and Price in 1996 [27] and belongs to the family of nature inspired techniques. It is simple and easy to cope, perform well in the presence of local minima, and has more randomness as compared to GA and PSO. The flow diagram for DE is shown in Figure 3(b), while the algorithm steps in the form of pseudocode are given.

Step 1 (initialization). The initialization step is similar to the one developed for PSO in (10).

Step 2 (updating). In this step, all chromosomes (particles) from 1 to $N$ of the current generation "ge" are updated. Suppose that we select $i$ th chromosome, that is, $\mathbf{c}_{k}^{i, \text { ge }}$ from (7), where $i=1,2, \ldots, N$ and $k=1,2, \ldots, 3 \times P$ and "ge" represents the particular generation. Now the goal is to find 
TABLE 2: Estimation accuracy of 1-L-shape array for 2 sources.

\begin{tabular}{lccccrr}
\hline Scheme & $s_{1}$ & $s_{2}$ & $\alpha_{1}{ }^{\circ}$ & $\alpha_{2}{ }^{\circ}$ & $\beta_{1}{ }^{\circ}$ \\
\hline Desired values & 0.5000 & 2.0000 & 30.0000 & 70.0000 & 110.0000 & 109.0038 \\
PSO & 0.4951 & 2.0050 & 30.0061 & 70.0060 & 210.0000 \\
DE & 0.5022 & 1.9977 & 30.0044 & 69.0058 & 110.0044 & 210.0061 \\
PSO-PS & 0.5018 & 2.0019 & 29.9967 & 70.0036 & 110.0033 & 209.9955 \\
DE-PS & 0.4991 & 1.9988 & 30.0015 & 69.0082 & 110.0015 & 210.0033 \\
\hline
\end{tabular}

the chromosome of next generation, that is, $\mathbf{c}^{i, \mathrm{ge}+1}$, by using the following steps.

(a) Mutation. In this step, pick up any three different numbers (chromosomes) from 1 to $N$, that is, $\left(n_{1}, n_{2}, n_{3}\right)$, under the following conditions:

$$
1 \leq n_{1}, n_{2}, n_{3} \leq N
$$

where

$$
\begin{aligned}
& n_{i} \neq n_{k} \quad \forall i, k=1,2,3, \\
& n_{i} \neq i \quad \forall i=1,2,3 ;
\end{aligned}
$$

now,

$$
\mathbf{d}^{i, \mathrm{ge}}=\mathbf{c}^{n_{1}, \mathrm{ge}}+F\left(\mathbf{c}^{n_{2}, \mathrm{ge}}-\mathbf{c}^{n_{3}, \mathrm{ge}}\right)
$$

where " $F$ " is a constant whose values usually lie in the range 0.5 to 1 .

(b) Crossover. The crossover can be performed as

$$
\mathbf{o}_{k}^{i, \text { ge }}= \begin{cases}\mathbf{d}_{k}^{i, \text { ge }} & \text { if rand }() \leq \mathrm{CR} \text { or } k=k_{\text {rand }} \\ \mathbf{c}_{k}^{i, g e} & \frac{o}{w},\end{cases}
$$

where $0.5 \leq \mathrm{CR} \leq 1$ and $k_{\text {rand }}$ is between 1 and $3 * P$ chosen at random.

(c) Selection Operation. The selection operation for the chromosome of next generation is performed as

$$
\mathbf{c}^{i, \text { ge }+1}= \begin{cases}\mathbf{o}^{i, \mathrm{ge}} & \text { if } \operatorname{err}\left(\mathbf{o}^{i, \mathrm{ge}}\right)<\operatorname{err}\left(\mathbf{c}^{i, \mathrm{ge}}\right) \\ \mathbf{c}^{i, \mathrm{ge}} & \frac{o}{w},\end{cases}
$$

where $\operatorname{err}\left(\mathbf{c}^{i, \mathrm{ge} e}\right)$ and $\operatorname{err}\left(\mathbf{o}^{i, \mathrm{ge}}\right)$ are defined in (15). Repeat this for all chromosomes.

(I) $\operatorname{err}\left(\mathbf{c}^{i, \text { get1}}\right)<\varepsilon$ (where $\varepsilon$ is a very small positive number).

(II) Total number of generations has completed.

Else, go back to Step 2.

Step 3 (termination). The termination criterion of DE is based on the following results achieved.

Step 4 (hybridization). In this step, the best results achieved through DE are given to PS for further refinement.

Step 5 (storage). Store all the results for later in discussion and comparison.

\section{Results and Discussion}

In the first part of simulations, various results are compared to evaluate the estimation accuracy and reliability of PSO, PSOPS, DE, and DE-PS for the joint estimation of amplitudes and DOA (elevation and azimuth) of far filed sources impinging on 1-L- and 2-L-shape arrays. In the second part of simulation, the comparison is carried out with PM, which has used parallel shape array [16] and L-shape arrays [17]. We have used 60 particles and 60 generations for PSO and DE, respectively. Each result is averaged over 100 independent runs.

Case 1. In this case, the estimation accuracy of PSO, DE, PSOPS, and DE-PS is examined for 1-L- and 2-L-shape arrays without having any noise in the system. Two sources are considered which have amplitudes $\left\{s_{1}=0.5, s_{2}=2\right\}$ and $\operatorname{DOA}\left\{\alpha_{1}=30^{\circ}, \beta_{1}=110^{\circ}, \alpha_{2}=70^{\circ}, \beta_{2}=210^{\circ}\right\}$. The 1-L-shape array consists of 5 sensors; that is, 2 sensors are placed along both the subarrays, that is, along $x$-axis and along $z$-axis, while the reference sensor is common for them. As provided in Table 2, one can clearly observe that the estimation accuracy of DE and PSO has been increased when they are hybridized with PS. The accuracy of PSO alone is less than DE but as the PSO is hybridized with PS, the estimation accuracy of PSO becomes even better than DE. However, the more accurate scheme is DE hybridized with PS.

In Table 3, the estimation accuracy of the same four schemes is provided for 2-L-shape array. The 2-L-shape array consists of 4 sensors; that is, each subarray consists of 1 sensor while the reference sensor is common for them. As listed in Table 3, again the hybrid DE-PS approach created fairly accurate results as compared to the other three schemes. Similarly, the other hybrid approach (PSO-PS) produced the second best accurate results.

Now by comparing Tables 2 and 3, it can be deduced very easily that each scheme not only produced better estimation accuracy in case of 2-L-shape array but also requires less number of sensors as compared to 1-L-shape array.

Case 2. In this subsection, the estimation accuracy is discussed for 3 sources impinging on L-shape arrays. The 1-L- and 2-L-shape arrays are composed of 13 and 7 sensors, respectively. The desired values of amplitudes $\left\{s_{1}=\right.$ $\left.2, s_{2}=4, s_{3}=6\right\}$ and DOA are $\left\{\alpha_{1}=60^{\circ}, \beta_{1}=15^{\circ}, \alpha_{2}=\right.$ $\left.25^{\circ}, \beta_{2}=85^{\circ}, \alpha_{3}=40^{\circ}, \beta_{3}=170^{\circ}\right\}$. The estimation accuracy of DE and PSO increases when they are hybridized with PS as depicted in Tables 4 and 5 . 
TABLE 3: Estimation accuracy of 2-L shape array for 2 sources.

\begin{tabular}{|c|c|c|c|c|c|c|}
\hline Scheme & $s_{1}$ & $s_{2}$ & $\alpha_{1}{ }^{\circ}$ & $\alpha_{2}{ }^{\circ}$ & $\beta_{1}{ }^{\circ}$ & $\beta_{2}{ }^{\circ}$ \\
\hline Desired values & 0.5000 & 2.0000 & 30.0000 & 70.0000 & 110.0000 & 210.0000 \\
\hline PSO & 0.5036 & 1.9963 & 29.9950 & 70.0049 & 110.0050 & 210.0050 \\
\hline $\mathrm{DE}$ & 0.4964 & 2.0014 & 30.0034 & 69.9966 & 110.0034 & 209.9965 \\
\hline PSO-PS & 0.4998 & 1.9991 & 30.0022 & 70.0021 & 109.9978 & 209.9979 \\
\hline DE-PS & 0.5001 & 2.0001 & 29.9998 & 70.0001 & 110.0002 & 210.0001 \\
\hline
\end{tabular}

TABLE 4: Estimation accuracy for 3 sources using 1-L-shape array.

\begin{tabular}{lccccccccc}
\hline Scheme & $s_{1}$ & $s_{2}$ & $s_{3}$ & $\alpha_{1}{ }^{\circ}$ & $\alpha_{2}{ }^{\circ}$ & $\alpha_{3}{ }^{\circ}$ & $\beta_{1}{ }^{\circ}$ & $\beta_{2}{ }^{\circ}$ & $\beta_{3}{ }^{\circ}$ \\
\hline Desired values & 2.0000 & 4.0000 & 6.0000 & 60.0000 & 25.0000 & 40.0000 & 15.0000 & 85.0000 & 170.0000 \\
PSO & 2.1789 & 3.8209 & 5.8210 & 60.3843 & 24.6158 & 39.6156 & 15.3847 & 84.6157 & 169.6153 \\
DE & 1.9028 & 3.9026 & 6.0971 & 58.8028 & 25.1974 & 40.1973 & 15.1979 & 85.1972 & 169.8022 \\
PSO-PS & 2.0191 & 4.0192 & 6.0193 & 59.9264 & 24.9261 & 40.0738 & 14.9261 & 84.9263 & 170.0737 \\
DE-PS & 1.9931 & 3.9932 & 5.9932 & 60.0268 & 24.9733 & 39.9731 & 15.0269 & 85.0260 & 170.0272 \\
\hline
\end{tabular}

The best estimation accuracy is given by DE-PS for both L-shape arrays, while the second best result is given by the hybrid PSO-PS technique. Overall, 2-L-shape array produced better estimation accuracy as compared to 1-L-shape array for all techniques.

Case 3. In this case, 4 sources are considered that have desired amplitude and DOA values are $\left\{s_{1}=1, s_{2}=3, s_{3}=\right.$ $\left.5, s_{4}=7\right\},\left\{\alpha_{1}=30^{\circ}, \beta_{1}=40^{\circ}, \alpha_{2}=50^{\circ}, \beta_{2}=65^{\circ}, \alpha_{3}=\right.$ $\left.85^{\circ}, \beta_{3}=255^{\circ}, \alpha_{4}=70^{\circ}, \beta_{4}=315^{\circ}\right\}$. However, the 1-L- and 2-L-shape arrays consist of 15 and 10 sensors, respectively. The estimation accuracy of PSO and DE degraded due to increase of sources. Again, the best result is produced by DEPS. Moreover, each scheme produced better results using 2-Lshape array with less number of antenna sensors as compared to 1-L-shape array as listed in Tables 6 and 7.

Case 4. In this case, the convergence of each scheme is investigated for 2, 3, and 4 sources. For convergence, the number of times particular schemes able to get the desired goal is analyzed. The number of sensors, values of amplitudes, and DOA are kept the same for this simulation as in Case 1. As shown in Figure 4, the convergence of PSO and DE has increased when they are hybridized with PS for all number of sources in case of 1-L-shape array. Moreover, the DE-PS scheme got fairly good convergence rate compared to the other schemes. The second best convergence rate is achieved by the hybrid PSO-PS approach.

Similarly, for 2-L-shape array, the DE-PS scheme got best convergence, while the second best convergence rate is achieved by PSO-PS as shown in Figure 5. From comparison of Figures 4 and 5, one can easily verify that each scheme produced better convergence for 2 -L-shape array as compared to 1-L-shape array.

It is evident from Figures 4 and 5 that DE-PS and PSOPS schemes have performed better for both L-shape arrays as compared to the other schemes. So further investigation will be carried out to analyze the performance of DE-PS and PSOPS schemes.

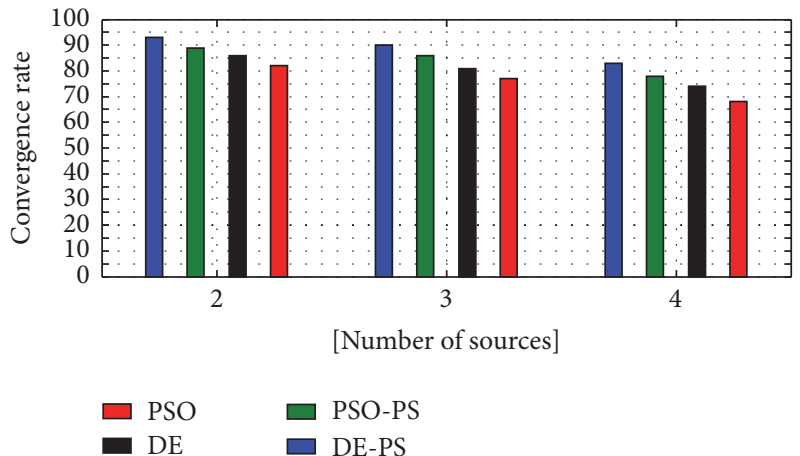

FIgURE 4: Performance analysis of convergence versus number of sources using 1-L-shape array.

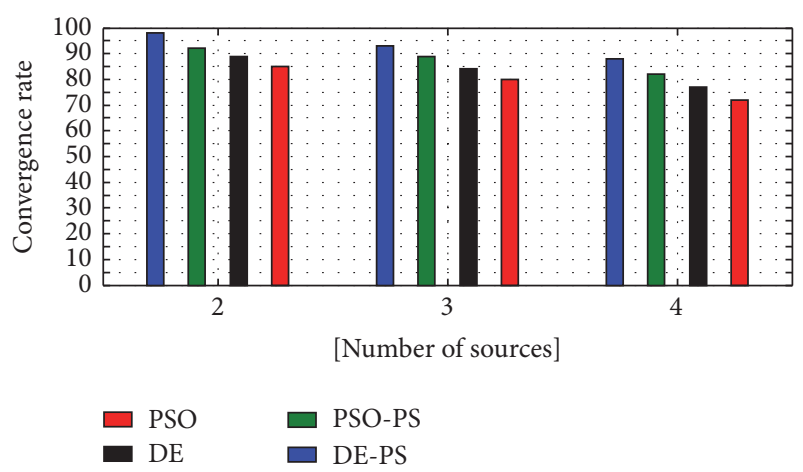

FIgURE 5: Performance analysis of convergence versus number of sources using 2-L-shape array.

Case 5. In this subsection, the proximity effects of elevation and azimuth angles are discussed using DE-PS and PSOPS schemes for both L-shape arrays. This experiment is performed for three sources. In Table 8 , the proximity of elevation angles for fixed amplitudes and azimuth angles is discussed. Due to proximity, the estimation accuracy and convergence rate of DE-PS and PSO-PS techniques degraded 
TABLE 5: Estimation accuracy for 3 sources using 2-L-shape array.

\begin{tabular}{lccccccccc}
\hline Scheme & $s_{1}$ & $s_{2}$ & $s_{3}$ & $\alpha_{1}{ }^{\circ}$ & $\alpha_{2}{ }^{\circ}$ & $\alpha_{3}{ }^{\circ}$ & $\beta_{1}{ }^{\circ}$ & $\beta_{2}{ }^{\circ}$ & $\beta_{3}{ }^{\circ}$ \\
\hline Desired values & 2.0000 & 4.0000 & 6.0000 & 60.0000 & 25.0000 & 40.0000 & 15.0000 & 85.0000 & 170.0000 \\
PSO & 1.8447 & 3.8445 & 6.1556 & 60.1791 & 25.1790 & 39.8207 & 15.1791 & 84.8206 & 170.1792 \\
DE & 2.0481 & 4.0480 & 6.0483 & 60.0977 & 24.9024 & 40.0979 & 14.9020 & 85.0983 & 169.9020 \\
PSO-PS & 2.0137 & 3.9862 & 6.0137 & 60.0328 & 25.0331 & 40.0330 & 15.0327 & 84.9670 & 170.0329 \\
DE-PS & 1.9980 & 4.0021 & 5.9980 & 59.9923 & 25.0077 & 39.9925 & 14.9925 & 85.0076 & 169.9926 \\
\hline
\end{tabular}

TABLE 6: Estimation accuracy for 4 sources using 1-L-shape array.

\begin{tabular}{lcccccccccccc}
\hline Scheme & $s_{1}$ & $s_{2}$ & $s_{3}$ & $s_{4}$ & $\alpha_{1}{ }^{\circ}$ & $\alpha_{2}{ }^{\circ}$ & $\alpha_{3}{ }^{\circ}$ & $\alpha_{4}{ }^{\circ}$ & $\beta_{1}{ }^{\circ}$ & $\beta_{2}{ }^{\circ}$ & $\beta_{3}{ }^{\circ}$ & $\beta_{4}{ }^{\circ}$ \\
\hline Desired values & 1.0000 & 3.0000 & 5.0000 & 7.0000 & 30.0000 & 50.0000 & 85.0000 & 70.0000 & 40.0000 & 65.0000 & 255.0000 & 315.0000 \\
PSO & 0.5211 & 2.5212 & 5.4792 & 7.4789 & 31.3274 & 51.3276 & 86.3282 & 69.6723 & 41.3276 & 66.3278 & 256.4006 & 316.4102 \\
DE & 1.2988 & 2.7009 & 5.2987 & 7.2990 & 29.0324 & 50.9678 & 85.9681 & 70.9679 & 40.9678 & 64.0320 & 256.0673 & 316.0874 \\
PSO-PS & 0.8143 & 2.8140 & 5.1858 & 7.1858 & 30.5741 & 50.5745 & 84.4252 & 70.5746 & 39.4252 & 65.5748 & 255.6775 & 315.8776 \\
DE-PS & 1.0989 & 2.9908 & 4.9010 & 7.0990 & 30.2468 & 50.2469 & 85.2471 & 69.7530 & 40.2470 & 65.2473 & 254.7431 & 315.3571 \\
\hline
\end{tabular}

TABLE 7: Estimation accuracy for 4 sources using 2-L-shape.

\begin{tabular}{lcccccccccccccc}
\hline Scheme & $s_{1}$ & $s_{2}$ & $s_{3}$ & $s_{4}$ & $\alpha_{1}{ }^{\circ}$ & $\alpha_{2}{ }^{\circ}$ & $\alpha_{3}{ }^{\circ}$ & $\alpha_{4}{ }^{\circ}$ & $\beta_{1}{ }^{\circ}$ & $\beta_{2}{ }^{\circ}$ & $\beta_{3}{ }^{\circ}$ & $\beta_{4}{ }^{\circ}$ \\
\hline Desired values & 1.0000 & 3.0000 & 5.0000 & 7.0000 & 30.0000 & 50.0000 & 85.0000 & 70.0000 & 40.0000 & 65.0000 & 255.0000 & 315.0000 \\
PSO & 0.7202 & 3.2799 & 5.2797 & 7.2711 & 31.1284 & 51.1285 & 86.1286 & 71.1283 & 41.1282 & 66.1284 & 256.1385 & 316.1369 \\
DE & 1.0988 & 3.0990 & 5.0989 & 6.9010 & 30.6656 & 49.3375 & 85.6654 & 70.6655 & 40.6656 & 65.6659 & 254.2342 & 315.6766 \\
PSO-PS & 1.0477 & 3.0479 & 4.9522 & 7.0480 & 30.2941 & 50.2942 & 84.7056 & 69.7060 & 40.2941 & 65.2946 & 255.2944 & 315.3041 \\
DE-PS & 1.0109 & 3.0111 & 5.0100 & 7.0112 & 30.0869 & 50.0870 & 85.0867 & 70.0869 & 40.0870 & 64.9125 & 254.9125 & 314.9082 \\
\hline
\end{tabular}

for both L-shape arrays but still the DE-PS technique is robust enough to produce fairly accurate results especially in case of 2-L-shape array.

In Table 9, the proximity of azimuth angles is discussed for fixed values of amplitudes and elevation. Again the DEPS technique acted well for the proximity of azimuth angles. It has shown good estimation accuracy and convergence rate especially for both L-shape arrays. Overall, the results of DEPS with 2-L-shape array are better as compared to 1-L-shape array and PSO-PS with both L-shape arrays.

Case 6. In this second part of simulations, the comparison of DE-PS using 2-L-shape array is carried out with PSO-PS using 2-L-shape array, PM using parallel shape array [16], and L-shape arrays [17]. Tables 10 and 11 listed the mean, variance, and standard deviations for PM using parallel shape array and L-shape array, respectively [16, 17], while Tables 12 and 13 provided the same calculation for PSO-PS and DEPS techniques, respectively, using 2-L-shape array. The PM uses 11 sensors for both parallel array and L-shape arrays configuration [16, 17], while for the PSO-PS and DE-PS schemes only 4 sensors are used in 2-L-shape array. The range of elevation angle is varied from $70^{\circ}$ to $90^{\circ}$ for fixed azimuth angle of $50^{\circ}$. This range of elevation angle has practical importance in wireless communication systems. The readers are encouraged to see [28] for further study. As obvious from Table 9, the PM method with parallel shape array has failed to produce accurate results as soon as the elevation angle is getting close to $90^{\circ}$ but the same PM method using 1-L-shape array configuration has got accurate results for the same range of elevation angle as listed in Table 10. However, at the same time, if we look at Tables 11 and 12, then one can observe that the DE-PS and PSO-PS schemes produced even better results for the same range of elevation angles by using 2-L-shape array configuration with less number of sensors. Overall the results of DE-PS are better.

Case 7. In this case, the Root Mean Square Error (RMSE) of DE-PS is compared with PSO-PS and PM using parallel shape array [16] and L-shape arrays [17]. Only one source is considered, which has elevation and azimuth angles $40^{\circ}$ and $65^{\circ}$, respectively. The range of signal-to-noise ratio (SNR) is taken from $5 \mathrm{~dB}$ to $30 \mathrm{~dB}$. The DE-PS technique maintains minimum RMSE for all values of SNR as depicted in Figure 6. The second best RMSE is maintained by PSO-PS using 2-Lshape arrays.

Case 8. In this subcase, the computational complexity of DE-PS is compared with PM using L-shape array [16]. The $\mathrm{PM}$ required total $\mathrm{O}(3 \times M \times T \times P)$ computations, where $M, P$, and $T$ represent total number of sensors, sources, and snapshots, respectively $[16,17]$. The main computations in DE-PS for 2-L-shape array involved the number of computations (multiplication) in fitness function $\mathrm{O}\left(\mathrm{N}^{2}(3+\right.$ $16 \times P))$ plus the multiplications required for mutation and cross-over which are approximately $2\left(16 \times N^{2}\right)$ where $N$ is the total number of particles. Moreover, the computations required for PS are approximately $(16 \times P)$. Therefore, the computational complexity of DE-PS for 2-L-shape array in terms of multiplication is almost $O\left(N^{2}(3+16 \times P)+2(16 \times\right.$ 
TABLE 8: Proximity effect of elevation angle for $s_{1}=1, s_{2}=3, s_{3}=5$ and $\beta_{1}=50^{\circ}, \beta_{2}=160^{\circ}, \beta_{3}=215^{\circ}$.

\begin{tabular}{|c|c|c|c|c|}
\hline Scheme & $\alpha_{1}^{\circ}$ & $\alpha_{2}{ }^{\circ}$ & $\alpha_{3}^{\circ}$ & $\%$ convergence \\
\hline Desired values & 30.0000 & 80.0000 & 50.0000 & 100 \\
\hline DE-PS (1-L) & 30.3843 & 80.3842 & 50.3844 & 90 \\
\hline PSO-PS (1-L) & 30.5132 & 80.5674 & 50.6312 & 88 \\
\hline DE-PS (2-L) & 30.1791 & 80.1790 & 50.1793 & 94 \\
\hline PSO-PS (2-L) & 30.3671 & 80.4101 & 50.3982 & 92 \\
\hline Desired values & 30.0000 & 65.0000 & 75.0000 & 100 \\
\hline DE-PS (1-L) & 30.3846 & 65.9832 & 75.9834 & 84 \\
\hline PSO-PS (1-L) & 30.5133 & 66.4378 & 76.6549 & 81 \\
\hline DE-PS (2-L) & 30.1792 & 65.4301 & 75.4302 & 92 \\
\hline PSO-PS (2-L) & 30.5134 & 66.1367 & 76.2136 & 88 \\
\hline Desired values & 30.0000 & 40.0000 & 50.0000 & 100 \\
\hline DE-PS (1-L) & 31.3965 & 41.4011 & 51.4013 & 70 \\
\hline PSO-PS (1-L) & 31.8709 & 41.9654 & 51.9987 & 64 \\
\hline DE-PS (2-L) & 30.7692 & 40.7694 & 50.7690 & 88 \\
\hline PSO-PS (2-L) & 31.3245 & 40.6788 & 50.9899 & 82 \\
\hline Desired values & 30.0000 & 35.0000 & 40.0000 & 100 \\
\hline DE-PS (1-L) & 32.3417 & 37.3518 & 42.3519 & 64 \\
\hline PSO-PS (1-L) & 34.1303 & 39.4321 & 43.9987 & 56 \\
\hline DE-PS (2-L) & 31.1105 & 36.1107 & 41.1105 & 82 \\
\hline PSO-PS (2-L) & 32.7989 & 37.0121 & 43.5467 & 76 \\
\hline
\end{tabular}

TABLE 9: Proximity effect of azimuth angles for $s_{1}=1, s_{2}=3, s_{3}=5$ and $\alpha_{1}=30^{\circ}, \alpha_{2}=50^{\circ}, \alpha_{3}=85^{\circ}$.

\begin{tabular}{|c|c|c|c|c|}
\hline Scheme & $\beta_{1}^{\circ}$ & $\beta_{2}^{\circ}$ & $\beta_{3}^{\circ}$ & $\%$ convergence \\
\hline Desired values & 15.0000 & 80.0000 & 230.0000 & 100 \\
\hline DE-PS (1-L) & 15.3841 & 80.3840 & 230.3845 & 90 \\
\hline PSO-PS (1-L) & 15.8865 & 80.7985 & 230.8891 & 88 \\
\hline DE-PS (2-L) & 15.1790 & 80.1791 & 230.1793 & 94 \\
\hline PSO-PS (2-L) & 15.4989 & 80.5110 & 230.5478 & 91 \\
\hline Desired values & 15.0000 & 80.0000 & 70.0000 & 100 \\
\hline DE-PS (1-L) & 15.3844 & 80.9830 & 70.9832 & 83 \\
\hline PSO-PS (1-L) & 15.5988 & 81.7896 & 71.9989 & 76 \\
\hline DE-PS (2-L) & 15.1792 & 80.4301 & 70.4302 & 91 \\
\hline PSO-PS (2-L) & 15.4989 & 81.1564 & 71.1211 & 87 \\
\hline Desired values & 60.0000 & 70.0000 & 80.0000 & 100 \\
\hline DE-PS (1-L) & 61.3966 & 71.4012 & 81.4014 & 72 \\
\hline PSO-PS (1-L) & 62.1456 & 62.5641 & 82.4538 & 65 \\
\hline DE-PS (2-L) & 60.7694 & 70.7696 & 80.7692 & 86 \\
\hline PSO-PS (2-L) & 61.5476 & 71.7873 & 81.6549 & 81 \\
\hline Desired values & 60.0000 & 65.0000 & 70.0000 & 100 \\
\hline DE-PS (1-L) & 62.3419 & 67.3519 & 72.3523 & 66 \\
\hline PSO-PS (1-L) & 64.8979 & 70.1653 & 74.9019 & 57 \\
\hline DE-PS (2-L) & 61.1103 & 66.1105 & 71.1104 & 80 \\
\hline PSO-PS (2-L) & 62.9109 & 68.9469 & 73.9381 & 74 \\
\hline
\end{tabular}

$\left.\left.N^{2}\right)+(16 \times P)\right)$. In the same way computations involved in DE-PS with 1-L-shape array are $\mathrm{ON}^{2}((3+20 \times P)+(20 \times P))$. The DE-PS for 2-L-shape array is computationally expensive as compared to DE-PS with 1-L-shape array, while it is less expensive as compared to PM.
Case 9. In Table 14, some general properties are listed for PM with parallel shape array, L-shape arrays, and DE-PS technique using 2-L-shape array. As presented in Table 8, the PM using I-L-shape array has an estimation failure problem in the range of elevation angles from $0^{\circ}$ to $20^{\circ}$. The second drawback 
TABLE 10: Means, variances, and standard deviations at $10 \mathrm{~dB}$ noise for different elevation angles and fixed azimuth $\beta=50^{\circ}$ by using PM with parallel shape array [16].

\begin{tabular}{lccc}
\hline$\alpha$ & Mean of $\widehat{\alpha}$ & Variance of $\alpha$ & Standard deviation of $\alpha$ \\
\hline $72^{\circ}$ & 72.0681 & 0.7021 & 0.8379 \\
$76^{\circ}$ & 74.9583 & 0.9895 & 0.9947 \\
$79^{\circ}$ & 77.8421 & 1.4082 & 1.1867 \\
$82^{\circ}$ & 80.4967 & 2.3482 & 1.5323 \\
$86^{\circ}$ & 83.3760 & 4.7215 & 2.1729 \\
$89^{\circ}$ & 84.7062 & 9.4567 & 3.0752 \\
\hline
\end{tabular}

TABLE 11: Means, variances, and standard deviations at $10 \mathrm{~dB}$ noise for different elevation angles and fixed azimuth $\beta=50^{\circ}$ by using PM with 1-L-shape array [17].

\begin{tabular}{lccc}
\hline$\alpha$ & Mean of $\widehat{\alpha}$ & Variance of $\alpha$ & Standard deviation of $\alpha$ \\
\hline $72^{\circ}$ & 72.3528 & 0.0151 & 0.1228 \\
$76^{\circ}$ & 76.2237 & 0.0111 & 0.1053 \\
$79^{\circ}$ & 79.0999 & 0.0068 & 0.0825 \\
$82^{\circ}$ & 82.0998 & 0.0037 & 0.0608 \\
$86^{\circ}$ & 86.0566 & 0.000632 & 0.0251 \\
$89^{\circ}$ & 89.0146 & 0.000371 & 0.0192 \\
\hline
\end{tabular}

TABLE 12: Means, variances, and standard deviations at $10 \mathrm{~dB}$ noise for different elevation angles and fixed azimuth $\beta=50^{\circ}$ by using PSO-PS with 2-L-shape array.

\begin{tabular}{cccc}
\hline$\alpha$ & Mean of $\hat{\alpha}$ & Variance of $\alpha$ & Standard deviation of $\alpha$ \\
\hline $72^{\circ}$ & 72.1354 & 0.002363 & $8.76 \times 10^{-03}$ \\
$76^{\circ}$ & 76.0003 & 0.003416 & $9.49 \times 10^{-03}$ \\
$79^{\circ}$ & 78.0075 & 0.008934 & $9.99 \times 10^{-03}$ \\
$82^{\circ}$ & 82.0046 & 0.006984 & $9.95 \times 10^{-03}$ \\
$86^{\circ}$ & 86.0097 & 0.007821 & $9.89 \times 10^{-03}$ \\
$89^{\circ}$ & 89.0078 & 0.008947 & $8.69 \times 10^{-03}$ \\
\hline
\end{tabular}

TABLE 13: Means, variances, and standard deviations at $10 \mathrm{~dB}$ noise for different elevation angles and fixed azimuth $\beta=50^{\circ}$ by using DE-PS with 2-L-shape array.

\begin{tabular}{lccc}
\hline$\alpha$ & Mean of $\hat{\alpha}$ & Variance of $\alpha$ & Standard deviation of $\alpha$ \\
\hline $72^{\circ}$ & 71.9998 & 0.00002415 & $4.91 \times 10^{-03}$ \\
$76^{\circ}$ & 76.0003 & 0.00001871 & $4.32 \times 10^{-03}$ \\
$79^{\circ}$ & 78.9996 & 0.00006854 & $8.27 \times 10^{-03}$ \\
$82^{\circ}$ & 81.9998 & 0.00003278 & $5.72 \times 10^{-03}$ \\
$86^{\circ}$ & 86.0006 & 0.00006132 & $7.83 \times 10^{-03}$ \\
$89^{\circ}$ & 89.0008 & 0.00001371 & $3.70 \times 10^{-03}$ \\
\hline
\end{tabular}

with 1-L-shape and parallel shape arrays is their limitation for elevation angles beyond $90^{\circ}$. Moreover, the parallel shape array has the pair matching problem. These limitations can be overcome by using PM with 2-L-shape arrays but it

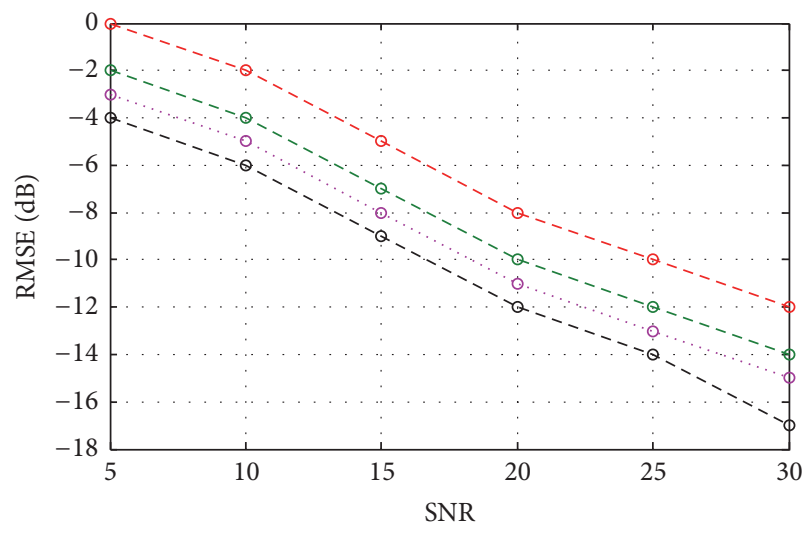

- - PM with parallel shape array [16]
$-\ominus-$ PM with L-shape array [17]
$\cdots \circ \cdot$ PSO-PS with 2-L-shape array
- - DE-PS with 2-L-shape array

FIGURE 6: Performance analysis of Root Mean Square Error versus SNR.

requires extra sensors and a large number of snapshots. It requires at least 200 snapshots that obviously increase the computational burdens. Therefore, the DE-PS technique removes the shortcomings of PM (1-L-shape and parallel shape) by using less number of sensors. The DE-PS technique also provides the estimation of source amplitude, which is very important parameter for accurate estimation of remote source. Moreover, it provides the appreciable decrease in the computational cost by requiring only a single snapshot to converge.

\section{Conclusion and Future Work Directions}

In this work, an efficient and robust approach has been proposed for joint estimation of amplitude and DOA of far-field sources using L-shape arrays. In this scheme, DE and PSO have been used as global optimizers, which were further assisted by PS as a rapid local search optimizer. We have demonstrated that the hybrid DE-PS approach performed considerably well as compared to the PSO, PSOPS, and DE techniques. It has been also shown that all the schemes performed well using 2-L-shape array as compared to 1-L-shape array. Moreover, the DE-PS scheme using 2L-shape array performed well as compared to PM using parallel shape array, as well as L-shape array. The simulation results are in complete conformation of adopted approach.

In future, one can check the same approach for sidelobe reduction and beam steering in adaptive beamforming

\section{Competing Interests}

The author declares that there is no conflict of interests regarding the publication of this paper. 
TABLE 14: Comparison among 2-L-shape array, parallel shape array [16], and L-shape arrays [17].

\begin{tabular}{lcccc}
\hline Property & Parallel shape array & 1-L-shape array & 2-L-shape array & 2-L-shape array \\
\hline Scheme used & PM & PM & PM & DE-PS \\
Range of elevation and azimuth angles & $\left(0^{\circ}, 90^{\circ}\right)$ and $\left(0^{\circ}, 360^{\circ}\right)$ & $\left(0^{\circ}, 90^{\circ}\right)$ and $\left(0^{\circ}, 360^{\circ}\right)$ & $\left(0^{\circ}, 180^{\circ}\right)$ and $\left(0^{\circ}, 360^{\circ}\right)$ & $\left(0^{\circ}, 180^{\circ}\right)$ and $\left(0^{\circ}, 360^{\circ}\right)$ \\
Number of sensors & 15 & 11 & 10 & 4 \\
Number of snapshots & 200 & 200 & 200 & 1 \\
Pair matching & Required & Not required & Not required & Not required \\
Failure estimation & From $70^{\circ}$ to $90^{\circ}$ & From $0^{\circ}$ to $20^{\circ}$ & No failure & No failure \\
Amplitude estimation & Cannot estimate & Cannot estimate & Cannot estimate & Yes, can estimate \\
\hline
\end{tabular}

\section{Acknowledgments}

This work is supported by Higher Education Commission (HEC) of Pakistan under Research Grant no. 21-323/SRGP/ $\mathrm{R} \& \mathrm{D} / \mathrm{HEC} / 2014$.

\section{References}

[1] Z. U. Khan, A. Naveed, I. M. Qureshi, and F. Zaman, "Independent null steering by decoupling complex weights," IEICE Electronics Express, vol. 8, no. 13, pp. 1008-1013, 2011.

[2] C.-C. Shen, "Maximum likelihood DOA estimation using particle swarm optimization under sensor perturbation conditions," Journal of Internet Technology, vol. 16, no. 5, pp. 847-855, 2015.

[3] Z. Zhang, J. Lin, and Y. Shi, "Application of artificial bee colony algorithm to maximum likelihood DOA estimation," Journal of Bionic Engineering, vol. 10, no. 1, pp. 100-109, 2013.

[4] R. Roy and T. Kailath, "ESPRIT-estimation of signal parameters via rotational invariance technique," IEEE Transactions on Acoustics, Speech, and Signal Processing, vol. 37, no. 7, pp. 984995, 1989.

[5] Y. D. Zhang, M. G. Amin, and B. Himed, "Joint DOD/DOA estimation in MIMO radar exploiting time-frequency signal representations," EURASIP Journal on Advances in Signal Processing, vol. 2012, article 102, pp. 1-10, 2012.

[6] M. F. Khan and M. Tufail, "Beamspace matrix pencil method for direction of arrival estimation," IEICE Electronics Express, vol. 6, no. 16, pp. 1168-1173, 2009.

[7] X. Zhong, A. B. Premkumar, and A. S. Madhukumar, "Particle filtering for acoustic source tracking in impulsive noise with alpha-stable process," IEEE Sensors Journal, vol. 13, no. 2, pp. 589-600, 2013.

[8] J.-C. Hung, "Modified particle swarm optimization structure approach to direction of arrival estimation," Applied Soft Computing Journal, vol. 13, no. 1, pp. 315-320, 2013.

[9] A. B. Gershman, M. Rübsamen, and M. Pesavento, "One- and two-dimensional direction-of-arrival estimation: an overview of search-free techniques," Signal Processing, vol. 90, no. 5, pp. 1338-1349, 2010.

[10] X. Zhong and A. B. Premkumar, "Particle filtering approaches for multiple acoustic source detection and 2-D direction of arrival estimation using a single acoustic vector sensor," IEEE Transactions on Signal Processing, vol. 60, no. 9, pp. 4719-4733, 2012.

[11] X. Zhong, A. B. Premkumar, and A. S. Madhukumar, "Particle filtering and posterior Cramér-Rao bound for 2-D direction of arrival tracking using an acoustic vector sensor," IEEE Sensors Journal, vol. 12, no. 2, pp. 363-377, 2012.
[12] H. Krim and M. Viberg, "Two decades of array signal processing research: the parametric approach," IEEE Signal Processing Magazine, vol. 13, no. 4, pp. 67-94, 1996.

[13] Q. Cheng and Y. Hua, "Further study of the pencil-MUSIC algorithm," IEEE Transactions on Aerospace and Electronic Systems, vol. 32, no. 1, pp. 284-299, 1996.

[14] Y. Hua, T. K. Sarkar, and D. D. Weiner, "An L-shaped array for estimating 2-D directions of wave arrival," IEEE Transactions on Antennas and Propagation, vol. 39, no. 2, pp. 143-146, 1991.

[15] V. S. Kedia and B. Chandna, "A new algorithm for 2-D DOA estimation," Signal Processing, vol. 60, no. 3, pp. 325-332, 1997.

[16] Y. Wu, G. Liao, and H. C. So, "A fast algorithm for 2-D directionof-arrival estimation," Signal Processing, vol. 83, no. 8, pp. 18271831, 2003.

[17] N. Tayem and H. M. Kwon, "L-shape 2-dimensional arrival angle estimation with propagator method," IEEE Transactions on Antennas and Propagation, vol. 53, no. 5, pp. 1622-1630, 2005.

[18] F. Zaman, I. M. Qureshi, J. A. Khan, and Z. U. Khan, "An application of artificial intelligence for the joint estimation of amplitude and two-dimensional direction of arrival of far field sources using 2-L-shape array," International Journal of Antennas and Propagation, vol. 2013, Article ID 593247, 10 pages, 2013.

[19] F. Zaman, I. M. Qureshi, F. Munir, and Z. U. Khan, "Fourdimensional parameter estimation of plane waves using swarming intelligence," Chinese Physics B, vol. 23, no. 7, Article ID 078402, 2014.

[20] F. Zaman and I. M. Qureshi, "5D parameter estimation of near-field sources using hybrid evolutionary computational techniques," The Scientific World Journal, vol. 2014, Article ID 310875, 11 pages, 2014.

[21] F. Zaman, I. M. Qureshi, A. Naveed, and Z. U. Khan, "Joint estimation of amplitude, direction of arrival and range of near field sources using memetic computing," Progress in Electromagnetics Research C, vol. 31, pp. 199-213, 2012.

[22] F. Zaman, I. M. Qureshi, A. Naveed, J. A. Khan, and R. M. A. Zahoor, "Amplitude and directional of arrival estimation: comparison between different techniques," Progress in Electromagnetics Research B, vol. 39, pp. 319-335, 2012.

[23] X. Zhang and J. Ma, "Pattern search methods for finite minimax problems," Journal of Applied Mathematics and Computing, vol. 32, no. 2, pp. 491-506, 2010.

[24] J. Kennedy and R. C. Eberhart, "Particle swarm optimization," in Proceedings of the IEEE International Conference on Neural Networks, pp. 1942-1948, IEEE Service Center, Perth, Australia, November 1995.

[25] Z. Yin, Z. Zong, H. Sun, Z. Wu, and Z. Yang, "A complexityperformance-balanced multiuser detector based on artificial 
fish swarm algorithm for DS-UWB systems in the AWGN and multipath environments," EURASIP Journal on Advances in Signal Processing, vol. 2012, article 229, 2012.

[26] O. Kaiwartya, S. Kumar, D. K. Lobiyal, P. K. Tiwari, A. H. Abdullah, and A. N. Hassan, "Multiobjective dynamic vehicle routing problem and time seed based solution using particle swarm optimization," Journal of Sensors, vol. 2015, Article ID 189832, 14 pages, 2015.

[27] R. Storn and K. Price, "Differential evolution-a simple and efficient heuristic for global optimization over continuous spaces," Journal of Global Optimization, vol. 11, no. 4, pp. 341359, 1997.

[28] N. A. H. M. Tayem, Direction of arrival angle estimation schemes for wirelerss communication systems [Ph.D. dissertation], Wichita State University, Wichita, Kan, USA, 2005. 


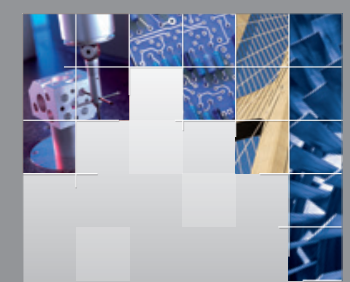

\section{Enfincering}
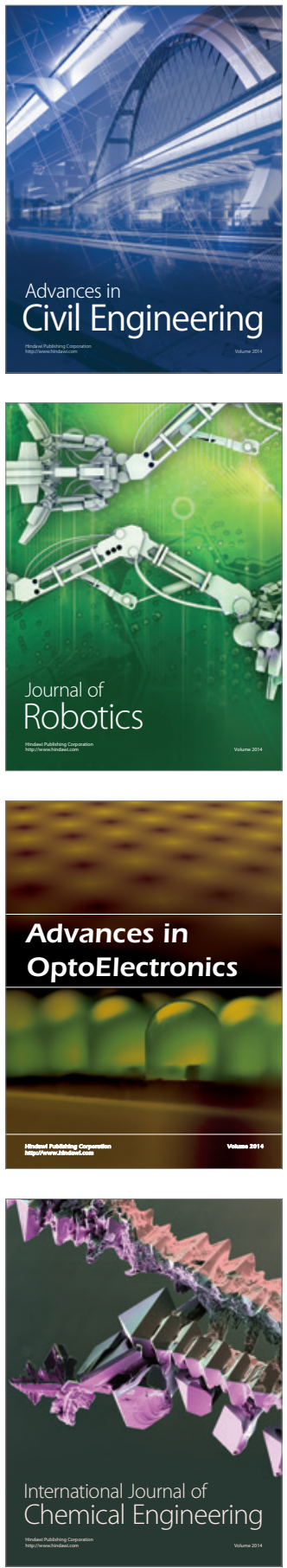

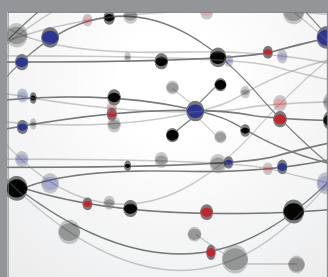

The Scientific World Journal

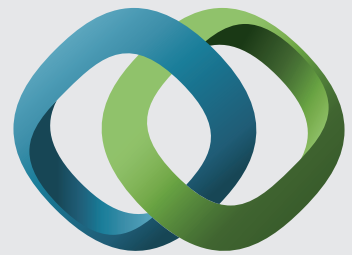

\section{Hindawi}

Submit your manuscripts at

https://www.hindawi.com
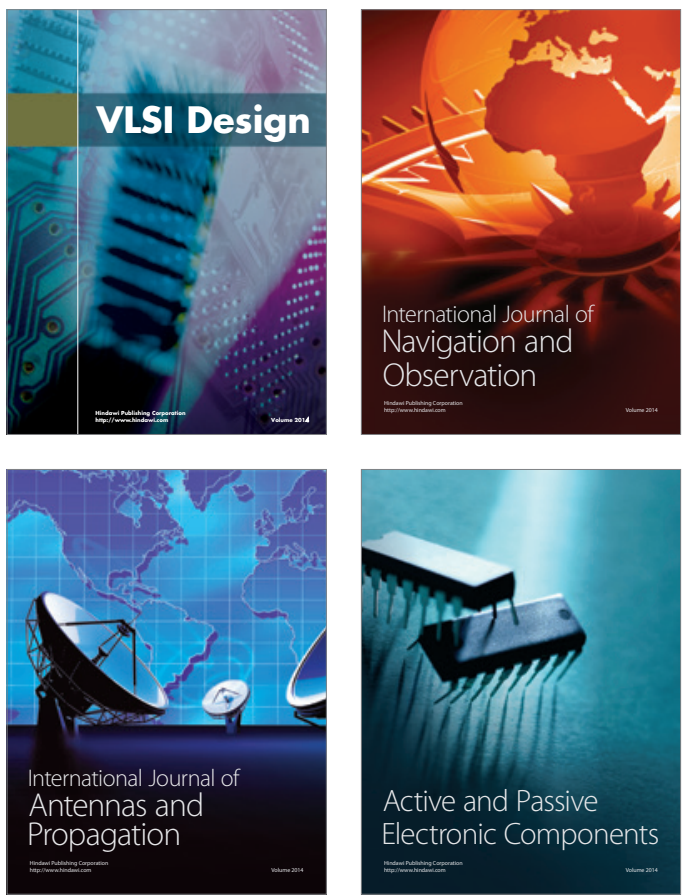
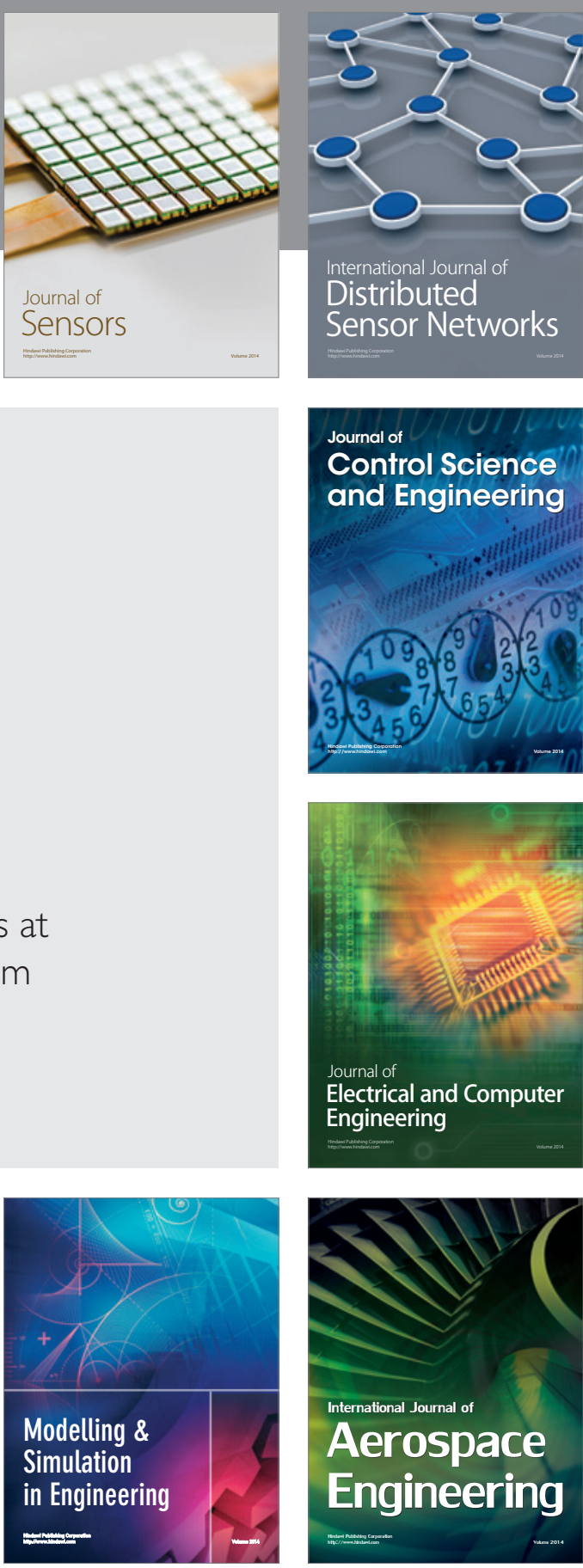

International Journal of

Distributed

Sensor Networks

$-$

Joumal of

Control Science

and Engineering
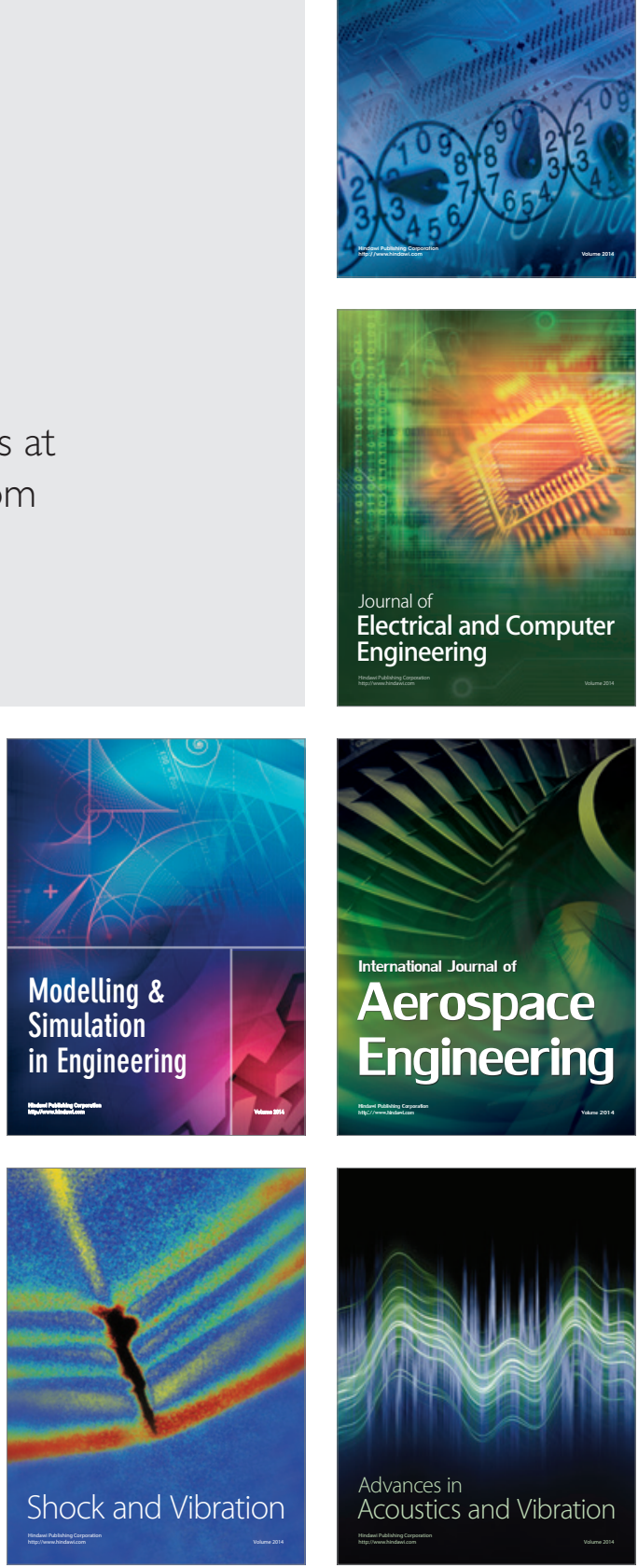